ISSN 1997-5902

\title{
Profil des lipides sériques des peuls adultes nomades du Nord-Bénin
}

\author{
Gomina Assoumanou Moutawakilou, Djaliri Kamal Dine et Akpona A. Simon \\ UER de Biochimie et de Biologie Moléculaire, Faculté de Médecine, Université de Parakou, BP : 123 Parakou \\ (République du Bénin)
}

Auteur correspondant : E-mail: elboutraguero@yahoo.fr ; Tel : +229 95966940

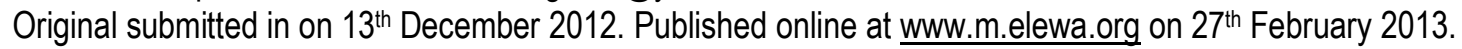

\section{RESUME}

Objectif : Décrire le profil des lipides sériques des sujets adultes peuls nomades du Nord-Bénin. Méthodes et résultats: II s'agissait d'une étude transversale à visée descriptive et analytique ayant concerné 58 sujets adultes peuls nomades et 61 non peuls. Les paramètres lipidiques ont été dosés par la méthode enzymatique conventionnelle. Les résultats ont montré que les valeurs moyennes de la cholestérolémie totale $(3,43 \pm 0,62$ $\mathrm{mmol} / \mathrm{L})$, de la cholestérolémie $\mathrm{HDL}(0,93 \pm 0,29 \mathrm{mmol} / \mathrm{L})$ et de la cholestérolémie $\mathrm{LDL}(1,84 \pm 0,61 \mathrm{mmol} / \mathrm{L})$ chez les sujets peuls étaient significativement inférieures à celles des sujets non peuls $(4,43 \pm 1,07 \mathrm{mmol} / \mathrm{L}$; $1,49 \pm 0,37 \mathrm{mmol} / \mathrm{L} ; 2,51 \pm 0,88 \mathrm{mmol} / \mathrm{L}$ respectivement).

Conclusion et application: Les peuls adultes nomades du Nord-Bénin ont un profil lipidique sérique différent de celui des autres ethnies, avec des concentrations en paramètres lipidiques sériques plus faibles. Nos résultats permettent de suggérer l'adoption du comportement alimentaire des peuls nomades du Nord-Bénin par la population générale afin de prévenir les dyslipidémies et certaines affections cardiovasculaires.

Mots clés : profil, lipides, sérum, peuls nomades, Bénin.

\section{Serum lipids profile of adult nomad Fulani populations in northern Benin ABSTRACT}

Objective: To describe the serum lipids profile of adult nomad Fulani subjects in northern Benin.

Methods and results: This work is about a cross-sectional descriptive and analytical study which involved 58 adult nomad subjects of Fulani origin and 61 adult subjects of non Fulani origin. Lipid parameters were analyzed through conventional enzymatic method. The results showed that mean values of total cholesterolemia ( $3.43 \pm 0.62 \mathrm{mmol} / \mathrm{L}), \mathrm{HDL}$ cholesterolemia $(0.93 \pm 0.29 \mathrm{mmol} / \mathrm{L})$ and LDL cholesterolemia $(1.84 \pm 0.61 \mathrm{mmol} / \mathrm{L})$ among Fulani subjects were significantly lower than that of non Fulani subjects $(4.43 \pm$ $1.07 \mathrm{mmol} / \mathrm{L} ; 1.49 \pm 0.37 \mathrm{mmol} / \mathrm{L} ; 2.51 \pm 0.88 \mathrm{mmol} / \mathrm{L}$ respectively).

Conclusion and application: The Fulani nomad adult populations living in northern Benin has a different serum lipids profile of that of other ethnic origins, with lower seric lipidic concentrations' parameters. These results allow us to suggest the adoption of northern Benin's Fulani nomads' dietary pattern for the rest of the Benin population in order to prevent dyslipidemias and other cardiovascular diseases.

Keywords: profile, lipids, serum, nomad Fulani, Benin. 


\section{INTRODUCTION}

La détermination des substrats lipoprotéiques est un des éléments de la première étape de la stratégie de prévention des maladies cardiovasculaires (ANAES, 2000). Elle permet d'évaluer les composantes lipidiques du sérum afin de déterminer une dyslipidémie (ANAES, 2000). Le bilan lipidique peut être réalisé dans plusieurs circonstances : dépistage systématique au cours d'une consultation, dépistage familial lorsqu'une anomalie lipidique héréditaire est retrouvée chez l'un des membres de la famille, certaines maladies notamment cardiovasculaires ou au cours de certains traitements, suivi thérapeutique (ANAES, 2000). En définitif l'évaluation des lipoprotéines sériques permet de prendre des mesures préventives (habitudes alimentaires, hygiène de vie) et/ou thérapeutiques.

Plusieurs facteurs peuvent influencer le profil lipoprotéique notamment les facteurs physiologiques (l'âge, le sexe, la grossesse), les facteurs génétiques, et les facteurs environnementaux tels que l'alimentation et la pratique du sport (Després et al ., 2000 ; Lindsquist et al ., 2000 ; Bamshad, 2005). Certaines affections agissent également sur le profil lipidique ; il s'agit du diabète sucré, de l'hypertension artérielle ou des troubles métaboliques (ANAES, 2000). Miljkovic et al. (2006) ont montré une différence de profil lipoprotéique entre les européens, les noirs américains et les noirs caraïbéins. Pour certains auteurs, les noirs américains ont de faibles concentrations de triglycérides (TG), de fortes concentrations de cholestérol HDL (C-HDL) et de faibles concentrations de cholestérol LDL (C-LDL) (Tyroler et al ., 1980 ; Lloyd-Jones et al ., 2010). Pour d'autres, les concentrations du cholestérol total (CT) dans les races blanche et noire tendent à être semblables alors que la concentration en C-HDL est plus élevée dans la race noire (Freedman et al ., 1990 ; Miljkovic et al ., 2006). Selon les travaux de

\section{MATERIEL ET METHODES}

Cadre d'étude: Cette étude a eu pour cadre, les communes de Gogounou et de Parakou en République du Bénin.

Matériel : Les réactifs utilisés dans le cadre de ce travail étaient : des kits de dosage du cholestérol, lot R276010,
Glueck et al . (1984) aux États-Unis d'Amérique, la proportion du CT /C-HDL est plus basse dans la race noire que dans la race blanche et la concentration en C-HDL est plus élevée chez les sujets Noirs américains par rapport aux sujets Blancs américains. De la même façon, les concentrations plasmatiques en TG sont logiquement inférieures chez les sujets Noirs américains comparés aux sujets Blancs américains (Wilson et al ., 1983). La population noire d'origine africaine vivant au Royaume Uni a une concentration plasmatique en TG inférieure d'une part et une concentration plasmatique en CHDL élevée d'autre part, comparativement aux britanniques de race blanche (Cappuccio et al 1997). Au Bénin, en dehors des travaux de Capo-Chichi (1992) sur le profil lipidique de référence du noir béninois et de Akpona et al . (1995 et 2009) sur les dyslipidémies et risque athérogène chez le diabétique et sur les valeurs de référence des apoprotéines, il n'existe pas de données sur les paramètres biochimiques lipidiques chez les peuls nomades qui sont pourtant très remarquables par leur mode de vie. En effet, ces peuls nomades pratiquent souvent presque le lacto-végétarisme naturel sans prétention idéologique ou religieuse. La viande de bœuf est particulièrement prohibée. Avec l'islam qu'ils pratiquent pour la majorité, les boissons alcoolisées ne sont pas consommées et leurs repas est en moyenne d'un plat unique par jour (Breton, 2008). Les peuls nomades ont alors un mode de vie qui se démarque des autres groupes ethniques et ayant pour principale activité l'élevage. De plus ils sont souvent physiquement maigres et rarement obèses. Un tel mode de vie des peuls nomades n'influencerait-il pas les concentrations de leurs paramètres lipidiques sériques?

L'objectif de cette étude était de décrire le profil des lipides sériques des sujets adultes peuls nomades du Nord-Bénin.

réf 230006 ; des kits de dosage des TG, lot R216010, réf 314005 ; des kits de précipitation des lipoprotéines de basse densité, lot $A 82420$, réf HDLc 5080 ; et du sérum de contrôle, lot 151340-01. Tous ces réactifs étaient de marque Spectrum.L'appareillage était constitué d'un 
spectrophotomètre de marque ERBA-chem-7 mannheim, d'une centrifugeuse ROTOFI×32 de marque Hettich et d'un bain marie de marque Sélecta $P$.

\section{Méthodes}

Type et période d'étude: II s'est agi d'une étude transversale à visée descriptive et analytique qui s'est déroulée du 1er septembre au 30 décembre 2011.

Population d'étude: La population d'étude était constituée de sujets peuls et de sujets non peuls. Ces sujets ont été sélectionnés selon nos critères d'inclusion, après un consentement éclairé lu et approuvé. Les sujets du groupe peul ont été sélectionnés dans trois campements peuls (Mondorou, Bagou peul, Kali peul) de la commune de Gogounou tandis que les sujets du groupe non peul ont été sélectionnés dans la commune de Parakou. Ont été inclus dans notre étude, les sujets des deux sexes, âgés de 18 à 65 ans, en bonne santé apparente et ayant consenti librement d'y participer. Ont été non inclus dans ce travail, les sujets diabétiques ou souffrant d'une affection métabolique quelconque, hypertendus, en dysthyroïdie, alcooliques, tabagiques, sous hormonothérapie, sous traitement médicamenteux susceptible de perturber le métabolisme des lipides et les femmes enceintes.

Échantillonnage: Nous avons effectué un sondage aléatoire à deux degrés. Le premier degré a consisté à choisir les quartiers de ville ou campements peuls ; cela a été fait par tirage au sort sans remise parmi les quartiers de Parakou d'une part et les campements peuls de Gogounou d'autre part. Le second degré a concerné le choix des sujets qui répondaient à nos critères d'inclusion. Toute personne répondant à ces critères d'inclusion et qui était présente le jour de la collecte des données était systématiquement prise en compte. Cette technique d'échantillonnage nous a permis de sélectionner 119 sujets répartis comme suit : 58 sujets ( 24 hommes et 34 femmes) pour le groupe peul et 61 sujets ( 29 hommes et 32 femmes) pour le groupe non peul.

Variables étudiées : Ont été prises en compte, l'âge, le sexe, l'indice de masse corporelle (IMC), les concentrations sériques de CT, de C-HDL, de C-LDL, de TG et l'indice d'athérogénicité (IA).

\section{RESULTATS}

Données anthropométriques des groupes étudiés : Les âges moyens des deux groupes de sujets n'étaient pas significativement différents $(p=0,875)$. Par contre il existe une différence significative entre les valeurs moyennes des IMC des deux groupes étudiés $(p=$
Collecte des données : Le recueil des données a été fait à l'aide d'un questionnaire écrit et administré. Chaque sujet de l'étude a bénéficié de la prise de son poids, de sa taille et d'un prélèvement de sang veineux en vue du dosage des paramètres lipidiques.

Prélèvements sanguins et traitement des échantillons de sang : Les prélèvements sanguins ont été effectués le matin chez les sujets à jeun depuis au moins 12 heures d'horloge. Quatre (4) $\mathrm{ml}$ de sang veineux ont été prélevés dans un tube sec chez chaque sujet. Les échantillons de sang ainsi obtenus chez les sujets peuls dans les campements de la commune de Gogounou ont été centrifugés à 4000 tours/minute pendant 5 minutes au laboratoire du Centre de Santé Communal de Gogounou, puis le sérum décanté a été congelé $\left(-5^{\circ} \mathrm{C}\right)$ et acheminé 48 heures après au laboratoire de biochimie du Centre Hospitalier Départemental du Borgou (CHD/B). Les échantillons de sang prélevés dans la commune de Parakou ont été acheminés le même jour au laboratoire de biochimie du $\mathrm{CHD} / \mathrm{B}$ et centrifugés comme décrit plus haut, en vue du dosage des paramètres lipidiques.

Dosage des paramètres lipidiques : Le dosage du CT a été effectué par la méthode enzymatique en point final au cholestérol oxydase (MacLachlan et al ., 2000) ; celui dosage des TG au glycérol phosphate oxydase (Solena, 2000) . Le dosage du C-HDL a été effectué par la méthode de précipitation à l'acide phosphotungstique en présence d'ions magnésiums (Warnick et al ., 1979). La concentration sérique de C-LDL et l'IA ont été déterminés par calcul.

Analyse statistique : Les données ont été analysées à l'aide du logiciel SPSS (version17). La concentration de C-LDL a été déterminée par calcul en utilisant la formule de Friedewald et al . (1972) qui n'est pas applicable lorsque la triglycéridémie est supérieure à $4,5 \mathrm{mmol} / \mathrm{L}$. L'IA a été obtenu à l'aide de la formule suivante: $I A=$ CT / C-HDL (Després et al ., 2000). Les résultats ont été présentés sous forme de moyennes avec leur écart-type et les valeurs extrêmes. L'analyse de la variance (ANOVA) affiné par le test $t$ de Student à permis de comparer les moyennes entre la population du groupe peul et celle du groupe non peul. La différence était significative pour un p inférieur à 0,05 .

0,000). Dans le groupe peul, la sex-ratio (hommes/femmes) était de 0,70 alors qu'elle était de 0,90 dans le groupe non peul. Le tableau 1 montre les caractéristiques anthropométriques de ces deux groupes. 
Gomina et al.... J. Appl. Biosci. 2013. Profil des lipides sériques des peuls adultes nomades du NordBenin

Tableau 1 : Données anthropométriques des groupes étudiés

\begin{tabular}{|c|c|c|c|c|c|c|c|}
\hline & \multicolumn{3}{|c|}{ Peuls } & \multicolumn{3}{|c|}{ Non peuls } & \multirow[t]{2}{*}{$p$} \\
\hline & Masculin & Féminin & Total & Masculin & Féminin & Total & \\
\hline Nombre de sujets & 24 & 34 & 58 & 29 & 32 & 61 & \\
\hline $\begin{array}{l}\text { Age moyen, années } \\
\text { ( } m \pm E T)\end{array}$ & $\begin{array}{c}39,25 \pm \\
12,84\end{array}$ & $\begin{array}{c}36,25 \pm \\
10,13\end{array}$ & $38 \pm 11$ & $\begin{array}{c}41,66 \pm \\
12,50\end{array}$ & $\begin{array}{c}36,97 \pm \\
11,64\end{array}$ & $39 \pm 12$ & 0,875 \\
\hline $\begin{array}{l}\text { Ages extrêmes, } \\
\text { années }\end{array}$ & $19-65$ & $18-62$ & $18-65$ & $22-62$ & $23-62$ & $22-62$ & \\
\hline $\begin{array}{l}\text { IMC moyen, } \mathrm{kg} / \mathrm{m}^{2} \\
(\mathrm{~m} \pm \mathrm{ET})\end{array}$ & $\begin{array}{c}18,79 \pm \\
2,90\end{array}$ & $\begin{array}{c}19,32 \pm \\
2,85\end{array}$ & $\begin{array}{c}19,10 \pm \\
2,86\end{array}$ & $\begin{array}{c}25,59 \pm \\
3,02\end{array}$ & $\begin{array}{c}29,80 \pm \\
5,79\end{array}$ & $\begin{array}{c}25,83 \pm \\
5,13\end{array}$ & 0,000 \\
\hline $\begin{array}{l}\text { IMC extrêmes, } \\
\mathrm{kg} / \mathrm{m}^{2}\end{array}$ & $\begin{array}{l}13,39- \\
23,59\end{array}$ & $\begin{array}{l}14,45- \\
25,70\end{array}$ & $\begin{array}{l}13,39- \\
25,70\end{array}$ & $\begin{array}{l}19,83- \\
30,42\end{array}$ & $\begin{array}{l}21,25- \\
44,28\end{array}$ & $\begin{array}{l}19,83- \\
44,28\end{array}$ & \\
\hline
\end{tabular}

$\mathrm{IMC}$ : indice de masse corporelle $; \mathrm{m} \pm \mathrm{ET}:$ moyenne \pm écart-type

Le profil des lipides sériques : Les valeurs moyennes du CT, du C-HDL et du C-LDL étaient significativement différentes entres les deux groupes de sujets $(p=0,000$; 0,$027 ; 0,000$ respectivement). Par contre il n'existe pas de différence significative entre les valeurs moyennes des
TG et de l'IA des deux groupes étudiés $(p=0,436 ; 0,737$ respectivement). Les valeurs moyennes et extrêmes des paramètres lipidiques des deux groupes de sujets ont été résumées dans le tableau 2.

Tableau 2 : Valeurs des paramètres lipidiques des groupes étudiés

\begin{tabular}{ll|c|c|c}
\hline & & Peuls & Non peuls & p \\
\hline Nombre de sujets & & 58 & 61 & \\
\hline CT, mmol/L & $\mathbf{m} \pm \mathbf{E T}$ & $3,43 \pm 0,62$ & $4,43 \pm 1,07$ & 0,000 \\
& Extrêmes & $2,47-4,79$ & $2,88-6,45$ & \\
\hline C-HDL, mmol/L & $\mathbf{m} \pm \mathbf{E T}$ & $0,93 \pm 0,29$ & $1,49 \pm 0,37$ & 0,027 \\
& Extrêmes & $0,64-1,93$ & $0,85-2,01$ & \\
\hline C-LDL, mmol/L & $\mathbf{m} \pm \mathbf{E T}$ & $1,84 \pm 0,61$ & $2,51 \pm 0,88$ & 0,000 \\
& Extrêmes & $0,41-3,09$ & $0,35-3,86$ & \\
\hline TG, $\mathbf{m m o l} / \mathbf{L}$ & $\mathbf{m} \mathbf{E T}$ & $1,00 \pm 0,49$ & $0,93 \pm 0,53$ & 0,436 \\
& Extrêmes & $0,53-2,22$ & $0,22-2,23$ & \\
\hline IA & $\mathbf{m} \pm \mathbf{E T}$ & $3,11 \pm 0,83$ & $3,06 \pm 0,78$ & 0,737 \\
& Extrêmes & $1,56-7,10$ & $1,66-6,08$ & \\
\hline
\end{tabular}

CT : cholestérol total ; C-HDL : cholestérol HDL ; C-LDL : cholestérol LDL; TG: triglycérides; IA: indice d'athérogénicité

Comparaison des paramètres lipidiques par sexe et par groupe de sujets : Chez les sujets de sexe féminin, les moyennes de la cholestérolémie totale, de la cholestérolémie HDL et de la cholestérolémie LDL étaient significativement différentes entre les sujets peuls et non peuls (respectivement $p=0,000 ; 0,015 ; 0,001$ ). II existe une différence significative entre les valeurs moyennes de l'IA chez les sujets peuls et non peuls de sexe masculin $(p=0,001)$ d'une part et d'autre part entre les sujets masculins et féminins du groupe peul $(p=$ 0,000 ). Le tableau 3 montre les valeurs moyennes des paramètres lipidiques par sexe et par groupe de sujets. 
Gomina et al.... J. Appl. Biosci. 2013. Profil des lipides sériques des peuls adultes nomades du NordBenin

Tableau 3 : Valeurs moyennes des paramètres lipidiques par sexe et par groupe de sujets

\begin{tabular}{|c|c|c|c|c|c|c|c|c|}
\hline \multirow[b]{3}{*}{$\begin{array}{l}\text { Nombre de } \\
\text { sujets }\end{array}$} & \multicolumn{2}{|c|}{ Peuls } & \multirow[t]{2}{*}{$p$} & \multicolumn{2}{|c|}{ Non peuls } & \multirow[t]{2}{*}{$p$} & \multicolumn{2}{|c|}{$\begin{array}{l}\text { Significativité entre } \\
\text { groupes d'étude }\end{array}$} \\
\hline & Masculin & Féminin & & Masculin & Féminin & & Masculin & Féminin \\
\hline & 24 & 34 & & 29 & 32 & & & \\
\hline $\mathrm{CT}, \mathrm{mmol} / \mathrm{L}$ & $\begin{array}{c}3,40 \pm \\
0,60\end{array}$ & $\begin{array}{c}3,46 \pm \\
0,64\end{array}$ & 0,476 & $\begin{array}{c}4,52 \pm \\
1,20\end{array}$ & $\begin{array}{l}4,34 \pm \\
0,93\end{array}$ & 0,593 & 0,125 & 0,000 \\
\hline $\begin{array}{l}\text { C-HDL, } \\
\mathrm{mmol} / \mathrm{L}\end{array}$ & $\begin{array}{c}0,98 \pm \\
0,24\end{array}$ & $\begin{array}{c}0,87 \pm \\
0,33\end{array}$ & 0,652 & $\begin{array}{l}1,46 \pm \\
0,35\end{array}$ & $\begin{array}{c}1,51 \pm \\
0,38\end{array}$ & 0,635 & 0,622 & 0,015 \\
\hline $\begin{array}{l}\text { C-LDL, } \\
\mathrm{mmol} / \mathrm{L}\end{array}$ & $\begin{array}{c}1,86 \pm \\
0,61\end{array}$ & $\begin{array}{c}1,82 \pm \\
0,61\end{array}$ & 0,545 & $\begin{array}{c}2,60 \pm \\
1,00\end{array}$ & $\begin{array}{c}2,42 \pm \\
0,75\end{array}$ & 0,417 & 0,622 & 0,001 \\
\hline $\mathrm{TG}, \mathrm{mmol} / \mathrm{L}$ & $\begin{array}{c}1,08 \pm \\
0,49\end{array}$ & $\begin{array}{c}0,92 \pm \\
0,49\end{array}$ & 0,751 & $\begin{array}{c}0,95 \pm \\
0,57\end{array}$ & $\begin{array}{c}0,91 \pm \\
0,49\end{array}$ & 0,131 & 0,227 & 0,922 \\
\hline IA & $\begin{array}{c}3,58 \pm \\
0,99\end{array}$ & $\begin{array}{c}2,78 \pm \\
0,48\end{array}$ & 0,000 & $\begin{array}{c}3,15 \pm \\
0,77\end{array}$ & $\begin{array}{c}2,98 \pm \\
0,78\end{array}$ & 0,400 & 0,001 & 0,216 \\
\hline
\end{tabular}

CT : cholestérol total ; C-HDL : cholestérol HDL ; C-LDL : cholestérol LDL; TG: triglycérides; IA: indice d'athérogénicité

\section{DISCUSSION}

Nous avons déterminé à travers une étude transversale descriptive et analytique le profil des lipides sériques des peuls adultes nomades du Nord-Bénin. La détermination des apoprotéines aurait rendu le travail exhaustif. Toutefois les résultats obtenus permettent de contribuer à l'établissement de bases de données des paramètres biologiques des peuls nomades du Nord-Bénin. Les âges moyens des sujets peuls de notre d'étude $(39,25 \pm 12,84$ ans pour les hommes et $36,25 \pm 10,13$ pour les femmes) sont supérieurs à ceux rapportés par Després et al . (2000) chez une population noire américaine $(32,7 \pm$ 11,9 ans pour les hommes et $31,71 \pm 11,1$ ans pour les femmes). La sex-ratio rapportée par les mêmes auteurs pour cette même population $(0,65)$ est superposable à celle trouvée dans notre travail $(0,70)$. Miljkovic-Gacic et al . (2010) avaient obtenu un âge moyen de 42,7 $\pm 16,8$ ans et une sex-ratio de 0,65 pour une population américaine d'ancêtres africains. Ces résultats ne sont pas différents de ceux des sujets peuls de notre étude (38 \pm 11 ans avec une sex-ratio de 0,70 ). La sex-ratio de 0,70 et 0,90 respectivement pour le groupe peul et le groupe non peul s'explique par le fait qu'au Nord-Bénin où nous avons réalisé cette étude, les femmes étaient beaucoup plus consentantes à participer à l'étude que les hommes. L'IMC moyen des sujets peuls de notre étude était inférieur à celui obtenu par Riediger et al . (2011) dans une population canadienne $\left(30 \mathrm{~kg} / \mathrm{m}^{2}\right)$. Les résultats obtenus par Després et al . (2000) $\left(22,76 \pm 8,9 \mathrm{~kg} / \mathrm{m}^{2}\right.$ chez les hommes et $29,66 \pm 9,7 \mathrm{~kg} / \mathrm{m}^{2}$ chez les femmes) pour la race blanche sont également supérieures à ceux des sujets peuls de notre étude. Ces résultats pourraient s'expliquer par la différence probable des habitudes alimentaires entre les sujets peuls et ceux du groupe non peul d'une part et entre les sujets peuls et ceux canadiens ou encore ceux de la race blanche d'autre part. En effet, les différents sujets sélectionnés dans ces différents groupes ne sont pas soumis aux mêmes exigences quotidiennes et n'ont pas les mêmes habitudes alimentaires. Lindsquist et al . (2000) ont montré aux USA qu'il existe des différences ethniques concernant certains paramètres anthropométriques comme l'IMC.

Nos résultats ont montré que la cholestérolémie totale chez les sujets peuls était inférieure à celle des sujets du groupe non peul aussi bien chez les hommes que chez les femmes. Cette différence était significative entre les deux groupes de sujets sans distinction de sexe $(3,48 \pm$ $0,64 \mathrm{mmol} / \mathrm{L}$ pour les sujets peulhs et $4,41 \pm 1,05 \mathrm{mmol} / \mathrm{L}$ pour les sujets du groupe non peul) avec $p=0,000<$ $5 \%$. Au niveau des hommes la différence n'était pas significative $(p=0,72)$ contrairement aux femmes pour les quelles elle était significative $(p=0,000<5 \%)$. Les résultats obtenus par Riediger et al . (2011) dans une population canadienne $(5,0 \pm 1,2 \mathrm{mmo} / \mathrm{L}$ pour les hommes et 4,8 $\pm 1,1 \mathrm{mmol} / \mathrm{L}$ pour les femmes) sont supérieurs aux nôtres. II en est de même des résultats de Després et al . (2000) aux USA pour la race noire $(4,39 \pm 0,93 \mathrm{mmol} / \mathrm{L}$ pour les hommes et $4,16 \pm 0,77$ 
$\mathrm{mmol} / \mathrm{L}$ pour les femmes) d'une part et pour la race blanche $(4,54 \pm 1,00 \mathrm{mmol} / \mathrm{L}$ pour les hommes et $4,42 \pm$ $0,89 \mathrm{mmo} / / \mathrm{L}$ pour les femmes) d'autre part. Nos résultats sont également inférieurs à ceux rapportés par Woo \& Lam (1990) dans une étude sur une population chinoise $(5,11 \pm 1,11 \mathrm{mmol} / \mathrm{L}$ pour les hommes et $5,79 \pm 1,02$ $\mathrm{mmol} / \mathrm{L}$ pour les femmes).

La concentration en cholestérol HDL chez les sujets peuls était inférieure à celle des sujets du groupe non peul aussi bien chez les hommes que chez les femmes dans notre étude. Nos résultats sont inférieurs à ceux rapportés par Woo \& Lam (1990) dans une population chinoise $(1,27 \pm 0,40 \mathrm{mmol} / \mathrm{L}$ pour les hommes et 1,51 \pm $0,36 \mathrm{mmol} / \mathrm{L}$ pour les femmes). Ils sont également inférieurs à ceux trouvés par Riediger et al . (2011) dans une population canadienne $(1,2 \pm 0,3 \mathrm{mmol} / \mathrm{L}$ pour les deux sexes). Després et al . (2000) avaient trouvé dans une population noire au USA un résultat similaire au nôtre pour les hommes $(1,00 \pm 0,34 \mathrm{mmol} / \mathrm{L})$ et supérieur pour les femmes $(1,12 \pm 0,27 \mathrm{mmol} / \mathrm{L})$.

Les moyennes de la cholestérolémie LDL des sujets peuls et non peuls étaient significativement différentes $(p$ $=0,000$ ) dans notre travail. Nos résultats sont inférieurs à ceux trouvés par Woo \& Lam (1990) dans une population chinoise $(3,11 \pm 1,03 \mathrm{mmol} / \mathrm{L}$ pour les hommes et $3,49 \pm$ $1,30 \mathrm{mmol} / \mathrm{L}$ pour les femmes). De même, Després et al . (2000) aux USA avaient obtenu des valeurs supérieures aux nôtres dans une population noire $(3,06 \pm 0,85$ $\mathrm{mmol} / \mathrm{L}$ pour les hommes et $2,92 \pm 0,77 \mathrm{mmol} / \mathrm{L}$ pour les femmes). Dans une étude réalisée sur une population canadienne, Riediger et al . (2011) avaient obtenu des valeurs de 2,9 $\pm 0,9 \mathrm{mmol} / \mathrm{L}$ pour les hommes et 2,60 \pm $0,9 \mathrm{mmol} / \mathrm{L}$ pour les femmes, résultats supérieurs aux nôtres. Les moyennes de la triglycéridémie des sujets peuls et non peuls n'étaient pas significativement différentes $(p=0,436)$ dans notre travail. Nos résultats sont inférieurs à ceux obtenus dans une étude réalisée sur une population chinoise $(1,98 \pm 1,56 \mathrm{mmol} / \mathrm{L}$ pour les hommes et 1,68 $\pm 1,68 \mathrm{mmol} / \mathrm{L}$ pour les femmes) par Woo \& Lam (1990). Des taux de triglycérides sériques supérieurs aux nôtres ont été rapportés par Riediger et al . (2011) au Canada (2,3 $\pm 2,5 \mathrm{mmol} / \mathrm{L}$ pour les hommes et $2,1 \pm 2 \mathrm{mmol} / \mathrm{L}$ pour les femmes) et par Després et al . (2000) aux USA, dans une population noire (1,54 \pm $0,89 \mathrm{mmol} / \mathrm{L}$ pour les hommes et $1,19 \pm 0,53 \mathrm{mmol} / \mathrm{L}$ pour les femmes). L'IA chez les sujets peuls de sexe féminin est inférieur à celui rapporté par Hunter et al . (2009) chez les femmes noires américaines (3,80 \pm $1,2)$ d'une part et chez les femmes blanches américaines d'autre part $(4,7 \pm 1,4)$. Després et al . (2000) aux USA avaient trouvé des résultats supérieurs aux nôtres dans une population de race noire $(4,68 \pm 1,58$ pour les hommes et $3,84 \pm 1,02$ pour les femmes). En général, la différence de concentration en substrats lipidiques sériques entre les deux groupes de sujets étudiés était significative: cholestérolémie totale $(p=0,000)$, cholestérolémie HDL $(p=0,027)$, cholestérolémie LDL ( $p$ $=0,000$ ). . Ces concentrations des paramètres lipidiques sériques faibles des peuls nomades du Nord-Bénin comparativement au groupe non peul multiethnique peuvent s'expliquer par deux principaux facteurs que sont : les facteurs génétiques d'une part et les facteurs environnementaux d'autre part. Nos résultats sont en accord avec ceux rapportés par plusieurs études où il a été montré une variabilité du profil lipidique sérique d'une race à une autre ou encore d'une ethnie à une autre (Glueck et al ., 1984 ; Freedman et al ., 1990; Linn et al ., 1991 ; Morrison et al ., 1998). Plusieurs études ont montré que les facteurs génétiques peuvent influencer le profil lipidique sérique. Després et al . (2000) ont rapporté cette différence du profil lipidique sérique entre la race blanche et la race noire. II est de même pour Miljkovic et al. (2006) qui ont abouti aux mêmes conclusions au cours d'une étude sur les déterminants génétiques de 33 lipoprotéines d'une population américaine d'ancêtres africains. De nombreuses études ont également montré l'influence des facteurs environnementaux sur le profil lipidique sérique (Woo \& Lam, 1990 ; Bamshad, 2005 ; Lohmueller et al ., 2006). Ces facteurs environnementaux regroupent l'alimentation, l'inactivité ou la prise d'alcool. Dans le présent travail, l'origine ethnique et les habitudes alimentaires des deux groupent étudiés diffèrent. 


\section{CONCLUSION}

La présente étude a décrit le profil des lipides sériques sur un échantillon de 119 sujets adultes répartis en deux groupes, un groupe de 61 sujets non peuls multiethnique et un groupe de 58 sujets peuls nomades du Nord-Bénin. Nos résultats montrent une différence dans plusieurs paramètres lipidiques sériques entre les deux groupes de sujets. Ainsi les sujets peuls nomades ont significativement des valeurs basses de concentrations sériques en CT, C-HDL et C-LDL par rapport aux sujets non peuls. En revanche la triglycéridémie et I'IA ne

\section{REFERENCES}

Agence National d'Accréditation et d'Evaluation de la Santé (ANAES), 2000. Modalités de dépistage et de diagnostic biologique des dyslipidémies en prévention primaire. ANAES / Service des recommandations et références professionnelles 70:38-48.

Akpona SA, Iwole S, Kora I, Lebreton P, Josse R, 1995. Dyslipidémie et risque athérogène au sein d'une population à risque à Cotonou. Méd. Afr. Noire 42(3) : 145-151.

Akpona AS, Gomina Assoumanou M, Soumanou MM, Adegbindin R, Abdoulaye I, 2009. Valeurs de référence de la lipoparticule (a) et de certaines apoprotéines chez le béninois par la technique d'électrophorèse en gel d'agarose. Rev.CAMES-Série A $8: 72-75$.

Bamshad M, 2005. Genetic influences on health: does race matter? JAMA 294: 937-946.

Breton $R, 2008$. Cartes de la répartition géographique des Peuls. Atlas des minorités dans le monde $978:$ : 7467-10917.

Capo-Chichi GC. 1992. Détermination du profil lipidique de référence du noir béninois. Thèse Pharma (Dakar), 61pp.

Cappuccio FP, Cook DG, Atkinson RW, Strazzullo P, 1997. Prevalence, detection, and management of cardiovascular risk factors in different ethnic groups in south London. Heart 78:555-563.

Després JP, Couillard C, Gagnon J, Bergeron J, Arthur S, James S, 2000. Race, Visceral Adipose Tissue, Plasma Lipids, and Lipoprotein Lipase Activity in Men and Women. The Health, Risk Factors, Exercise Training, and Genetics (HERITAGE) Family Study. Arterioscler Thromb Vasc Biol 20(8):1932-1938.doi:10.1161/01.ATV.20.8.1932.

Freedman DS, Strogatz DS, Eaker E, 1990. Differences between black and white men in correlates of diffèrent pas significativement entre les deux populations étudiées. Ces taux bas des lipides sériques et particulièrement du C-LDL pourraient élucider la rareté des vasculopathies athéromateuses chez les sujets peuls nomades du Nord-Bénin. Nos résultats permettent de suggérer l'adoption du comportement alimentaire des peuls nomades du Nord-Bénin par la population générale afin de prévenir les dyslipidémies et certaines affections cardiovasculaires.

high density lipoprotein cholesterol. Am J Epidemiol 132:656-669.

Friedewald WT, Levy RI, Fredrickson DS, 1972. Estimation of the concentration of low density lipoprotein cholesterol in plasma without use of ultracentrifuge. Clin Chem 18: 499-502.

Glueck CJ, Gartside P, Laskarzewski PM, Nestlerode CS, 1984. High-density lipoprotein cholesterol in blacks and whites: potential ramifications for coronary heart disease. Am Heart J 108: 815826.

Lindsquist $\mathrm{CH}$, Gower BA, Goran MI, 2000. Role of dietary factors in ethnic difference in early risk of cardiovascular disease and type 2 diabetes. Am J Clin Nutr 71:725-732.

Linn S, Fulwood R, Carroll M, Crowther NJ, 1991. Serum total cholesterol: HDL cholesterol ratios in U.S. white and black adults by selected demographic and socioeconomic variables (HANES II). Am J Public Health 181:1038-1043.

Lohmueller KE, Mauney MM, Reich D, Braverman JM, 2006. Variants associated with common disease are not unusually differentiated in frequency across populations. Am J Hum Genet 78:130 136.

Lloyd-Jones D, Adams RJ, Brown TM, Carnethon M, 2010. Heart disease and stroke statistics update: a report from the American Heart Association. Circulation 121:46-110

MacLachlan J, Wotherspoon AT, Ansell RO, Brooks CJ, 2000. Cholesterol oxidase: sources, physical properties and analytical applications. J Steroïd Biochem Mol Biol 72(5): 169-195.

Miljkovic G, Bunker $\mathrm{CH}$, Ferrell RE, Kammerer CM, Evans RW Wheeler VW, 2006. Lipoprotein subclass and particle size differences in AfroCaribbean, African Americans, and white 
Americans: associations with hepatic lipase gene variation. Metabolism 55: $96-102$.

Morrison JA, Barton BA, Biro FM, Sprecher DL, 1998. The conjoint trait of low high-density lipoprotein cholesterol and high triglycerides in adolescent black and white males. Metabolism 47: 514521.

Riediger ND, Bruce SG, Kue Young T, 2011. Cardiovascular Risk According to Plasma Apolipoprotein and Lipid Profiles in a Canadian First Nation. Prev Chronic Dis 8(1):1-8.

Solena M-L, 2000. Métabolisme des lipides et des lipoprotéines. In : Valdiguié $P$, editors. Biochimie clinique. Paris : cedex; pp168-186.

Tyroler HA, Glueck CJ, Christensen B, Kwiterovich JrPO, 1980. Plasma high-density lipoprotein cholesterol comparisons in black and white populations. The Lipid Research Clinics Program Prevalence Study. Circulation 62:99107.

Warnick GR, Cheung MC, Albers JJ, 1979. Comparison of current methods for high-density lipoprotein cholesterol quantification. Clin Chem 25(4):596604.

Wilson PWF, Savage DD, Castelli WP, Davis CE, Tyroler HA, 1983. HDL-cholesterol in a sample of black adults: the Framingham Minority Study. Metabolism 32:328-332.

Woo J. and Lam CW, 1990. Serum lipid profile in an elderly Chinese. Arterioscler Thromb Vasc Biol 10:1097-1101 doi :10.1161/01.ATV.10.6.1097. 